\title{
Comparative study of in vitro prepared Rose Bengal Plate Test (RBPT) antigen with commercially available antigens
}

\author{
Mujeeb ur Rhaman ${ }^{1}$, Amir Ullah ${ }^{2}$, Junaid Ali Shah ${ }^{3}$ \\ ${ }^{I}$ College of Life Sciences, Northwest University, Xi'an, Shaanxi province, China. \\ ${ }^{2}$ Department of Laboratory Medicine, Southern Medical University, Guangzhou, China. \\ ${ }^{3}$ College of Life Sciences, Jilin University, China Changchun city, jilin Province
}

Received: 2020-06-18.

Accepted: 2020-08-03

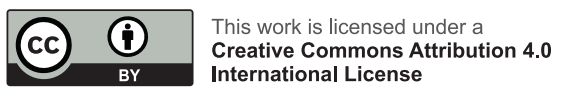

J Clin Med Kaz 2020; 5(59):46-50

Corresponding author:

Mujeeb ur Rahman.

Email: mujeeb@stumail.nwu.edu.cn

\section{Abstract}

Background and aim: Brucellosis is one of the world most common zoonotic diseases. The current study was aimed to prepare the Rose Bengal Plate Test (RBPT) antigen for the diagnosis of brucellosis and to determine its specificity and sensitivity.

Material and methods: The Rose Bengal plate test antigen prepared from Brucella abortus (B. abortus) strain 99 was compared with two commercial Rose Bengal Plate Test antigens and its specificity and sensitivity are determined.

Results: The were Rose Bengal plate test and I-ELISA result show that the in vitro antigen was superior to RBPT antigen University Diagnosis Laboratory (UDL) Lahore Pakistan, and RBPT antigen Veterinary Laboratory Agency (VLA) UK. Out of 196 samples analyzed by in vitro RBPT antigen, RBPT antigen (UDL), RBPT antigen (VLA), and an indirect enzyme-linked immunosorbent assay (I-ELISA) $56.63 \%, 53.57 \%$, $41.84 \%, 35.71 \%$ were found B. abortus positively. The sensitivity calculated for the in vitro RBPT antigen was 96.62, while RBPT antigen (UDL) and RBPT antigen (VLA) were 89.77, 63.91 correspondingly. However, the specificity of the in vitro RBPT antigen was lower (77.57\%), than the commercial RBPT antigen (VLA) (79.79\%).

Conclusions: A very sensitive and low-cost in vitro RBPT antigen compared to commercial RBPT was magnificently developed in the current study. It was determined that the in vitro RBPT antigen could substitute the available commercial RBPT antigen, which is comparatively expensive and less sensitive in the detection of brucellosis. Therefore, it is concluded that the in vitro RBPT antigen could be used for epidemiological surveillance of brucellosis.

Key words: brucella, antigen, diagnosis, serology, animals

\section{Introduction}

Brucellosis is a major globally re-emerging zoonosis, which mostly affects domestic animals such as, cattle, goat, sheep, swine, buffalo, and dogs caused by Brucella species [1, 2]. They are Gram-negative, non-hemolytic, non-motile, non-spore-forming and facultative intra-cellular living, coccobacilli. However, Brucellae demonstrate a preference for a certain host, e.g. Brucella Melitensis prefers small ruminants, B. abortus bovine, the transmission of cross-species occurs when various animals are in near contact with one another [38]. Brucellosis is endemic in several geographical regions like the Middle East and Mediterranean region, Mexico, parts of Central and South America and South Asian countries including India, Pakistan, Srilanka and China [9-11]. Although in very few economically developed countries this disease is controlled, it is still an issue that causes a significant globally economic loss [12]. Fetal membrane retention (FMR) and last trimester abortion are the characteristic signs in female animals whereas epididymitis and orchitis are common in males but the infection may remain asymptomatic and the infected animals may remain undiagnosed [13]. Through milk and vaginal secretions, the infected animals shed bacteria 
in the environment [14]. In animals, brucellosis is typically transmitted either through direct contact or by the ingestion of contaminated water or feed, while in humans this usually occurs through the ingestion of contaminated milk $[15,16]$. Human beings are accidental hosts for this infectious disease and can be prevented by eradicating the disease in animals, which often have close interaction with humans $[17,18]$.

The diagnosis of brucellosis verified by isolation which is the gold standard but this process is time-consuming, laborious, low sensitivity and additionally there is a high risk of infection. Hence, serological test including Rose Bengal Plate Test (RBPT), Enzyme-Linked Immunosorbent Assay (ELISA), Indirect Enzyme-Linked Immunosorbent Assay (iELISA) and Complement Fixation Test (CFT) is normally used for the diagnosis of Brucella [19-21]. As no serological test is $100 \%$ reliable, diagnosis is usually based on two or more test results.

Consequently, early testing is usually done using a screening test, which is a highly sensitive test and perhaps less specific. The screening tests are typically cost-effective, quick and easy to perform. If a positive reaction occurs during a screening test, a confirmatory test is carried out. The confirmatory test is a kind of test that offers good sensitivity but relatively higher test specificity, thus eliminating some false positives reaction. The majority of confirmatory tests are more complex and very expensive to carry out [22].

The Rose Bengal plate test is a rapid test that was originally developed for screening use in veterinary medicine but is now also used to diagnose human brucellosis [23,24]. Its high sensitivity, ease of use and affordable prices make it extremely common in hospital emergency departments for the diagnosis of febrile syndromes.

The present study aimed to develop RBPT antigens from Brucella abortus strain 99, compared with commercial PBPT antigens University Diagnosis Laboratory (UDL) Lahore, Pakistan and Veterinary Laboratory Agency (VLA) UK and finally, its specificity and sensitivity were determined.

\section{Material and methods}

This research study was conducted at Animal Sciences Institute, National Agriculture Research Center Islamabad, Pakistan from October 2015 to December 2016. Blood samples were collected from goats and sheep at the slaughterhouse in Islamabad. Approximately 5-7 $\mathrm{mL}$ of blood was obtained in a transparent tube without any anticoagulant and placed on ice immediately. All the specimens collected for animals had either aborted or reproductive disorder such as infertility. Samples were transported to the Animal Sciences Institute, bacteriology laboratory after proper labelling and sealing. The sera were isolated by centrifugation of blood samples at $1500 \mathrm{rpm}$ for 10 mins and preserved at $-20{ }^{\circ} \mathrm{C}$ for further analysis.

The culture of B. abortus strain 99 was inoculated on Tryptic Soy Agar (TSA) media for three days at $37^{\circ} \mathrm{C}$. Initially, the Gram stain and then Ziehl-Neelsen (Z-N) staining was carried out. Then, biochemical tests, such as Urease, Oxidase, Catalase, Indole production, Methyl Red, Simmon Citrate utilization and Voges-Proskauer tests were conducted for further confirmation.

The B. abortus strain 99 was used to prepare the RBPT antigen. The antigen was prepared following the previous literature by Office International des Epizooties (2009) [25]. Briefly, 4-5 colonies of B. abortus were inoculated into TSA broth media and incubated at $37{ }^{\circ} \mathrm{C}$ for 48 hours. The liquid media was centrifuged to get an isolated organism. The pellet was re-suspended in $0.5 \%$ phenol saline. The mixture was discarded, and the pellet was heated at $80^{\circ} \mathrm{C}$ for 90 minutes to kill bacteria and mixed with 1\% Rose Bengal. The suspension was stored at $4^{\circ} \mathrm{C}$.

The prepared antigen and serum were kept at room temperature $\left(22 \pm 4^{\circ} \mathrm{C}\right)$. Initially, $20 \mu \mathrm{l}$ serum was added on the white porcelain plate through a pipette. Then, $20 \mu \mathrm{l}$ of that antigen was mixed with the serum and shaken for four minutes. The data was recorded as positive after agglutination. The I-ELISA was used as a gold standard to determine the sensitivity and specificity of RBPT antigen.

\section{Data analysis}

The specificity and sensitivity were determined by using the following formula [26]:

Sensitivity $=\frac{\text { True Positive }}{\text { True Positive }+ \text { False negative }} \times 100$
Specificity $=\frac{\text { True Positive }}{\text { True Positive }+ \text { False negative }} \times 100$

\section{Results}

Brucella isolate was grown on selective Tryptic Soy Agar for morphological examination and incubated for 2-3 days. The Brucella colonies were characterized as smooth, glistening, pinpoint, bluish, honey-colored and translucent [27]. Microscopically, the culture smear appeared as gram-negative coccobacilli in Gram staining and red-stained coccobacilli in modified Z-N staining. Therefore, these finding shows the resemblance with bacteria characteristic of Brucella [28]. The isolate was positive to Oxidase, Catalase, and Urease tests and negative to Methyl Red, Indole production, Simmon Citrate utilization and Voges-Proskauer tests, these results were in agreement with the previous study [29]. The in vitro prepared RBPT antigen was tested by mixing $20 \mu \mathrm{l}$ of the sample with 20 $\mu 1$ of prepared antigen on a porcelain plate. In the presence of distinct agglutination the reaction was declared positive (Figure $1)$.

Figure 1 - Rose Bengal Plate Test. Figure A representing a strong agglutination reaction.

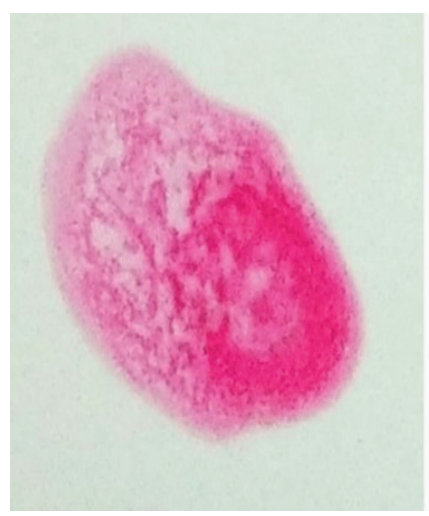

Positive Reaction

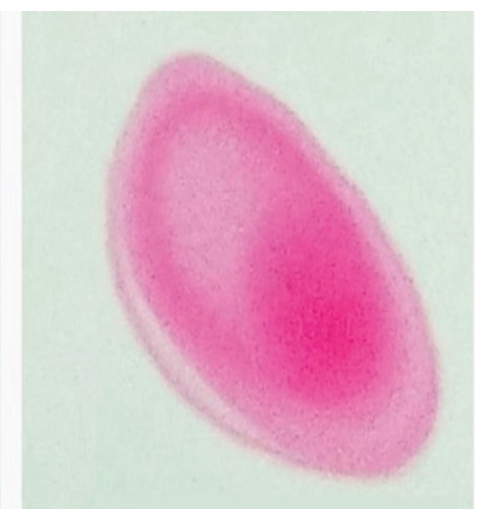

Negative Reaction
The in vitro RBPT antigen was compared with RBPT antigen (UDL), a total number of 196 samples were processed, and the result explored that in vitro RBPT antigen was 111 (RBPT+) and 85 were (RBPT-), while the RBPT antigen (UDL) showed that 105 were $(\mathrm{RBPT}+)$ and 91 were (RBPT-), as mentioned in Table 1. 

antigen (UDL)

\begin{tabular}{lllll}
\hline Sample & $\begin{array}{l}\text { In vitro } \\
\text { RBPT Ag }\end{array}$ & Percentage & $\begin{array}{l}\text { RBPT antigen } \\
\text { (UDL) }\end{array}$ & Percentage \\
RBPT (+) & 111 & 56.63 & 105 & 53.57 \\
RBPT (-) & 85 & 43.37 & 91 & 46.43 \\
Total & 196 & & 196 & \\
\hline
\end{tabular}

$\mathrm{Ag}=$ Antigen, $\mathrm{RBPT}=$ Rose Bengal plate test antigen, $\mathrm{UDL}=$ University Diagnosis

Laboratory, VLA=Veterinary Laboratory Agency

The in vitro RBPT antigen was further compared with the RBPT antigen (VLA), and the results showed that 82/196 were (RBPT+) and (RBPT-) was 114/196 (Table 2).

\section{Table 2}

Percentage of in vitro RBPT antigen and RBPT antigen (VLA)

\begin{tabular}{lllll} 
Sample & $\begin{array}{l}\text { In vitro } \\
\text { RBPT Ag }\end{array}$ & Percentage & $\begin{array}{l}\text { RBPT antigen } \\
\text { (VLA) }\end{array}$ & Percentage \\
\hline RBPT (+) & 111 & 56.63 & 82 & 41.84 \\
RBPT (-) & 85 & 43.37 & 114 & 58.16 \\
Total & 196 & & 196 & \\
\hline
\end{tabular}

Finally, in vitro RBPT antigen was compared with I-ELISA, the results were as follows; ELISA+ 73/196, and ELISA- 123/196 (Table 3).

\begin{tabular}{lllll}
\multicolumn{1}{c}{ Table 3} & \multicolumn{2}{c}{$\begin{array}{c}\text { Percentage of in vitro RBPT antigen and } \\
\text { I-ELISA }\end{array}$} & & \\
\hline Sample & $\begin{array}{l}\text { In vitro } \\
\text { RBPT Ag }\end{array}$ & Percentage & I-ELISA & Percentage \\
& 111 & 56.63 & 73 & 37.24 \\
RBPT (+) & 85 & 43.37 & 123 & 62.76 \\
RBPT (-) & 196 & & 196 & \\
Total & & & & \\
\hline
\end{tabular}

The sensitivity and specificity of in vitro RBPT antigen, RBPT antigen (UDL) and RBPT antigen (VLA) were tested. The results showed that the sensitivity of in vitro RBPT antigen was $96.62 \%$, which was higher than UDL $89.77 \%$, while the specificity was high potent in VLA $79.79 \%$ than in vitro RBPT Ag $77.57 \%$ (Table 4).

\begin{tabular}{llll}
\multicolumn{2}{c}{ Table 4} & \multicolumn{3}{l}{$\begin{array}{l}\text { Specificity and sensitivity of in vitro RBPT Ag, } \\
\text { RBPT antigen (UDL) and RBPT antigen (VLA) }\end{array}$} \\
\hline S. No & Antigens & Sensitivity (\%) & Specificity (\%) \\
1 & $\begin{array}{l}\text { In vitro RBPT } \\
\text { Ag }\end{array}$ & 96.62 & 77.57 \\
2 & $\begin{array}{l}\text { RBPT antigen } \\
\text { (UDL) }\end{array}$ & 89.77 & 73.83 \\
3 & $\begin{array}{l}\text { RBPT antigen } \\
\text { (VLA) }\end{array}$ & 63.91 & 79.79 \\
\hline
\end{tabular}

\section{Discussion}

Brucellosis is a zoonotic bacterial disease caused by Brucella genus. In the present study, RBPT antigen was prepared from B. abortus strain 99. There are many serological tests uses for the determination of brucellosis, but we prefer RBPT, which has considerably high sensitivity while I-ELISA used for the specificity of the brucellosis diagnosis [10]. The I-ELISA has been regarded as a gold standard by many researchers to compare the results for brucellosis diagnosis [30]. In this study, I-ELISA was used as a gold standard to calculate the specificity and sensitivity of in vitro RBPT antigen and two other commercial RBPT antigens.

A study showed that out of 856 sera, $31.66 \%$ was positive by CFT using commercial RBPT and in-house RBPT detected $30.84 \%$ positive animals respectively [31]. However, the current study indicates that out of 196 sera, $37.24 \%$ was found to be positive by I-ELISA, while the in vitro RBPT antigen, RBPT antigen (UDL) and RBPT antigen (VLA) detected 56.63\%, $53.57 \%, 41.83 \%$, positive animals, respectively. Moreover, we observed that 85 (in vitro RBPT), 91 (RBPT antigen (UDL), 114 (RBPT antigen (VLA) sera were negative with in vitro RBPT while some of them were positive with I-ELISA. The falsenegative result of the RBPT antigen could be the "Zoning effect" in acidified RBPT antigen [32]. An additional factor which may lead to false-negative result can be the outdated RBPT antigen. The sensitivity of the RBPT antigen could be lost during the storage process by the improper addition of reagents.

A study reported that the in-house RBPT was (85.24\%) sensitive as compared to commercial RBPT (78.59\%), but the commercial RBPT (97.77\%) was more specific than the inhouse RBPT $(94.36 \%)$ [31]. The present study explored that the in vitro antigen (96.62\%) was more sensitive compared to the RBPT antigen (UDL) (89.77\%) and RBPT antigen (VLA) (63.91\%). Nevertheless, the RBPT antigen (VLA) $(79.79 \%)$ is more specific than the in vitro RBPT antigen $(77.57 \%)$. However, we reported 111 (in vitro antigen), 105 (RBPT antigen (UDL) and 82 (RBPT antigen VLA) out of total sera samples were RBPT positive but some of them were I-ELISA negative. These false-positive results may be due to the vaccination of B. abortus strain 19 or exposure to a gram-negative organism having lipopolysaccharide (LPS) O-chain similar to Brucellae species, like E. coli O:157, V. cholerae O1, Y. enterocolitica O:9 and Salmonella group N (O:30) [33]. The current study suggested that the sensitivity of the in vitro antigen was more significant than previous studies, which were $85.2 \%$ and $90.1 \%$ respectively $[31,34]$. The possible reason may be the optimization methods achieved using international imported B. abortus serum to determine the specificity and sensitivity of developed in vitro antigen.

\section{Conclusion}

This study suggests that the in vitro RBPT antigen which is a low cost, rapid and has high sensitivity as compared to the commercial RBPT antigens. Finally, this diagnostic technique recommended replacing the commercially available RBPT antigen, which is relatively costly and less sensitive in the identification of brucellosis in sheep and goats.

Disclosures: There is no conflict of interest for all authors.

Acknowledgement: The authors are very thankful to the Animal Sciences Institute (ASI), National Agriculture Research Center (NARC) Islamabad, Pakistan, for their collaboration.

Funding: none 


\section{References}

1. Godfroid J, Scholz H, Barbier T, Nicolas C, Wattiau P, Fretin D, et al. Brucellosis at the animal/ecosystem/human interface at the beginning of the 21st century. Preventive veterinary medicine. 2011; 102(2):118-131. PubMed: https://www.ncbi.nlm.nih.gov/ pubmed/?term=. https://doi.org/10.1016/j.prevetmed.2011.04.007

2. Scholz HC, Revilla-Fernández S, Al Dahouk S, Hammerl JA, Zygmunt MS, Cloeckaert A, et al. Brucella vulpis sp. nov., isolated from mandibular lymph nodes of red foxes (Vulpes vulpes). International journal of systematic and evolutionary microbiology. 2016; 66(5):2090-2098. PubMed: https://www.ncbi.nlm.nih.gov/pubmed/?term=. https://doi.org/10.1099/ijsem.0.000998

3. Aparicio ED. Epidemiology of brucellosis in domestic animals caused by Brucella melitensis, Brucella suis and Brucella abortus. Rev Sci Tech. 2013; 32(1):53-60. PubMed: https://www.ncbi.nlm.nih.gov/pubmed/?term=. https://doi.org/10.20506/rst.32.1.2187

4. Saleem MZ, Akhtar R, Aslam A, Rashid MI, Chaudhry ZI, Manzoor MA, et al. Evidence of Brucella abortus in non-preferred caprine and ovine hosts by real-time PCR assay. Pak J Zool. 2019; 51:1187-1189. PubMed: https://www.ncbi.nlm.nih.gov/pubmed/?term=. https://doi.org/10.17582/journal.pjz/2019.51.3.sc3

5. Saeed U, Ali S, Khan TM, El-Adawy H, Melzer F, Khan AU, et al. Seroepidemiology and the molecular detection of animal brucellosis in Punjab, Pakistan. Microorganisms. 2019; 7(10):449. PubMed: https://www.ncbi.nlm.nih.gov/pubmed/?term=. https://doi.org/10.3390/ microorganisms 7100449

6. Jamil T, Melzer F, Khan I, Iqbal M, Saqib M, Hammad Hussain M, et al. Serological and molecular investigation of Brucella species in dogs in Pakistan. Pathogens. 2019; 8(4):294. PubMed: https://www.ncbi.nlm.nih.gov/pubmed/?term=. https://doi.org/10.3390/ pathogens 8040294

7. Fatima S, Khan I, Nasir A, Younus M, Saqib M, Melzer F, et al. Serological, molecular detection and potential risk factors associated with camel brucellosis in Pakistan. Tropical animal health and production. 2016; 48(8):1711-1718. PubMed: https://www.ncbi.nlm. nih.gov/pubmed/?term=. https://doi.org/10.1007/s11250-016-1148-9

8. Foster JT, Walker FM, Rannals BD, Hussain MH, Drees KP, Tiller RV, et al. African lineage Brucella melitensis isolates from Omani livestock. Frontiers in microbiology. 2018; 8:2702. PubMed: https://www.ncbi.nlm.nih.gov/pubmed/?term=. https://doi.org/10.3389/ fmicb.2017.02702

9. Norman FF, Monge-Maillo B, Chamorro-Tojeiro S, Pérez-Molina J-A, López-Vélez R. Imported brucellosis: a case series and literature review. Travel medicine and infectious disease. 2016; 14(3):182-199. PubMed: https://www.ncbi.nlm.nih.gov/pubmed/?term=. https:// doi.org/10.1016/j.tmaid.2016.05.005

10. Neha A, Kumar A, Ahmed I. Comparative efficacy of serological diagnostic methods and evaluation of polymerase chain reaction for diagnosis of bovine brucellosis. Iranian journal of veterinary research. 2017; 18(4):279. PubMed: https://www.ncbi.nlm.nih.gov/ pubmed/?term=.

11. Franc K, Krecek R, Häsler B, Arenas-Gamboa A. Brucellosis remains a neglected disease in the developing world: a call for interdisciplinary action. BMC public health. 2018; 18(1):1-9. PubMed: https://www.ncbi.nlm.nih.gov/pubmed/?term=. https://doi. org/10.1186/s12889-017-5016-y

12. Seleem MN, Boyle SM, Sriranganathan N. Brucellosis: a re-emerging zoonosis. Veterinary microbiology. 2010; 140(3-4):392-398. PubMed: https://www.ncbi.nlm.nih.gov/pubmed/?term=. https://doi.org/10.1016/j.vetmic.2009.06.021

13. Corbel MJ. Brucellosis in humans and animals: World Health Organization; 2006.

14. Akhtar S, Mirza M. Rates of seroconversion in the progeny of Brucella abortus seropositive and seronegative cattle and buffalo. Revue scientifique et technique (International Office of Epizootics). 1995; 14(3):711-718. PubMed: https://www.ncbi.nlm.nih.gov/ pubmed/?term=. https://doi.org/10.20506/rst.14.3.861

15. Dadar M, Shahali Y, Whatmore AM. Human brucellosis caused by raw dairy products: A review on the occurrence, major risk factors and prevention. International journal of food microbiology. 2019; 292:39-47. PubMed: https://www.ncbi.nlm.nih.gov/pubmed/?term=. https://doi.org/10.1016/j.ijfoodmicro.2018.12.009

16. Hakeem M, Saeed S. Brucellosis: A case report and literature review. J Postgrad Med Ed Res. 2019; 53:126-127. PubMed: https:// www.ncbi.nlm.nih.gov/pubmed/?term=. https://doi.org/10.5005/jp-journals-10028-1328

17. Young E, Hall W, Evans A, Brachman P. Bacterial infection of humans: epidemiology and control. 1998. PubMed: https://www.ncbi. nlm.nih.gov/pubmed/?term $=$.

18. 18. Rubach MP, Halliday JE, Cleaveland S, Crump JA. Brucellosis in low-income and middle-income countries. Current opinion in infectious diseases. 2013; 26(5):404. PubMed: https:/www.ncbi.nlm.nih.gov/pubmed/?term=. https://doi.org/10.1097/ QCO.0b013e3283638104

19. Glynn MK, Lynn TV. Zoonosis update. AVMA. 2008; 233:900-908. PubMed: https://www.ncbi.nlm.nih.gov/pubmed/?term=.

20. Muñoz P, Marín C, Monreal D, Gonzalez D, Garin-Bastuji B, Diaz R, et al. Efficacy of several serological tests and antigens for diagnosis of bovine brucellosis in the presence of false-positive serological results due to Yersinia enterocolitica O: 9. Clinical and Diagnostic Laboratory Immunology. 2005; 12(1):141-151. PubMed: https://www.ncbi.nlm.nih.gov/pubmed/?term=. https://doi. org/10.1128/CDLI.12.1.141-151.2005

21. Refai M. Incidence and control of brucellosis in the Near East region. Veterinary microbiology. 2002; 90(1-4):81-110. PubMed: https:// www.ncbi.nlm.nih.gov/pubmed/?term=. https://doi.org/10.1016/S0378-1135(02)00248-1

22. 22. Padilla PF, Nielsen K, Ernesto SL, Ling YW. Diagnosis of brucellosis. The Open Veterinary Science Journal. 2010; 4(1):46-60. PubMed: https://www.ncbi.nlm.nih.gov/pubmed/?term=. https://doi.org/10.2174/1874318801004010046

23. Morgan WB, MacKinnon D, Cullen G. Rose Bengal plate agglutination test in the diagnosis of brucellosis. Veterinary record. 1969. PubMed: https://www.ncbi.nlm.nih.gov/pubmed/?term=. https://doi.org/10.1136/vr.85.23.636

24. Oomen L, Waghela S. The rose bengal plate test in human brucellosis. Tropical and geographical medicine. 1974; 26(3):300-302. PubMed: https://www.ncbi.nlm.nih.gov/pubmed/?term=.

25. Alexander D. Newcastle, disease: OIE Terrestrial Manual 2009. Manual of diagnostic tests and vaccines for terrestrial animals. 2009:3. PubMed: https://www.ncbi.nlm.nih.gov/pubmed/?term=. 
26. Akhtar R, Chaudhry ZI, Shakoori A, Ahmad M, Aslam A. Comparative efficacy of conventional diagnostic methods and evaluation of polymerase chain reaction for the diagnosis of bovine brucellosis. Vet World. 2010; 3(2):53-56. PubMed: https://www.ncbi.nlm.nih.gov/ pubmed/?term $=$.

27. Abbas BA, Mohammed HQ, Tofah JA. Diagnostic Study on Brucella melitensis isolated from Human in Wassit province. AlMustansiriyah Journal for Pharmaceutical Sciences. 2012; 12(2):8-24. PubMed: https://www.ncbi.nlm.nih.gov/pubmed/?term=.

28. Gilligan P, York M, Baselski V, Carey R, Krisher K, Lovchik J, et al. Sentinel Laboratory Guidelines for Suspected Agents of Bioterrorism: Brucella Species. Washington, ASM Press. 2004; 1:4-10. PubMed: https://www.ncbi.nlm.nih.gov/pubmed/?term=.

29. Noviello S, Gallo R, Kelly M, Limberger RJ, DeAngelis K, Cain L, et al. Laboratory-acquired brucellosis. Emerging infectious diseases. 2004; 10(10):1848. PubMed: https://www.ncbi.nlm.nih.gov/pubmed/?term=. https://doi.org/10.3201/eid1010.040076

30. Zakaria AM. Comparative Assessment of Sensitivity and Specificity of Rose Bengal Test and Modified In-House ELISA by using IS711 Taqman Real Time PCR Assay as a Gold Standard for the Diagnosis of Bovine Brucellosis. Biomedical and Pharmacology Journal. 2018; 11(2):951-957. PubMed: https://www.ncbi.nlm.nih.gov/pubmed/?term=. https://doi.org/10.13005/bpj/1453

31. Shahaza O, Khairani-Bejo S, Zunita Z, Bahaman A. In-house Rose Bengal Plate Agglutination Test (RBPT) for a rapid diagnosis of brucellosis in goats in Malaysia. Int J Trop Med. 2009; 4(3):116-118. PubMed: https://www.ncbi.nlm.nih.gov/pubmed/?term=.

32. Saxena HM, Chothe S, Kaur P. Simple solutions to false results with plate/slide agglutination tests in diagnosis of infectious diseases of man and animals. MethodsX. 2015; 2:345-352. PubMed: https://www.ncbi.nlm.nih.gov/pubmed/?term=. https://doi.org/10.1016/j. mex.2015.08.001

33. Manishimwe R, Ntaganda J, Habimana R, Nishimwe K, Byukusenge M, Dutuze F, et al. Comparison between Rose Bengal Plate Test and competitive enzyme linked immunosorbent assay to detect bovine brucellosis in Kigali City, Rwanda. J Vet Sci Technol. 2015; 6:14. PubMed: https://www.ncbi.nlm.nih.gov/pubmed/?term=.

34. Yahaya SM, Khairani-Bejo S, Zunita Z, Omar MA, Bitrus AA. Development of an in-house Rose Bengal Plate test for diagnosis of brucellosis in goat. Malaysian Journal of Veterinary Research. 2016; 7(2):59-65. PubMed: https://www.ncbi.nlm.nih.gov/ pubmed/?term $=$. 